\title{
Tecnología Étnico-Digital: Recursos Didácticos Convergentes en el Desarrollo de Competencias Matemáticas en los Estudiantes de Zona Rural
}

\author{
Julia A. Ramón ${ }^{(1)}$ y Jesús Vilchez ${ }^{(2) \star}$ \\ (1) Colegio de Aplicación Rural, Univ. Hermilio Valdizán, Av. Universitaria 607, Huánuco-Perú \\ (e-mail: angelaramonortiz@gmail.com) \\ (2) Universidad Hermilio Valdizán, Fac. de Educación, Dpto. de Ciencias, Sr. de los Milagros Mz. D-4, Huánuco-Perú. \\ (e-mail: jjevilchez17@gmail.com) \\ * Autor a quien debe ser dirigida la correspondencia
}

Recibido Ago. 24, 2018; Aceptado Nov. 20, 2018; Versión final Dic. 17, 2018, Publicado Jun. 2019

\begin{abstract}
Resumen
Se analiza el nivel de influencia del uso de recursos étnico-digitales en el proceso de enseñanza-aprendizaje de las matemáticas en estudiantes del tercer grado de educación secundaria de zonas rurales de la provincia de Huánuco (Perú), orientado al logro de sus competencias matemáticas. El estudio se contextualiza en la modalidad de investigación-acción participativa, a través del diseño de ejecución concurrente. La implementación de la estrategia didáctica se realiza durante seis semanas de clases. La experiencia realizada permite observar, que el proceso de enseñanza-aprendizaje de la matemática contextualizada e interactiva mediado por los recursos del entorno y digitales, generan alta motivación y compromiso de los estudiantes con su aprendizaje; la misma, que se evidencia en la eficiencia mostrada en el proceso de construcción de conceptos, proceso de modelación y resolución de problemas matemáticos. Se concluye que la convergencia de la tecnología étnica y digital, dinamizan el proceso didáctico y favorecen el logro de aprendizajes significativos de los tópicos de la matemática por los estudiantes.
\end{abstract}

\section{Digital-Ethnic Technology: Converging Didactic Resources in the Development of Mathematical Competences in Rural Area Students}

\begin{abstract}
The level of influence of the use of ethno-digital resources in the teaching-learning process of mathematics in students of the third grade of secondary education in rural areas of the province of Huánuco is analyzed, oriented to the achievement of their mathematical competences. The study is contextualized in the modality of participatory action research, through the design of concurrent execution. The implementation of the teaching strategy is carried out during six weeks of classes. The experience made allows observing that the teachinglearning process of contextualized and interactive mathematics mediated by the environment and digital resources, generate high motivation and commitment of students with their learning; the same, which is evident in the efficiency shown in the process of concept construction, modeling process and solving mathematical problems. It is concluded that the convergence of ethnic and digital technology, energizes the didactic process and favor the achievement of significant learning of the topics of mathematics by students.
\end{abstract}

Keywords: ethnic technology; digital technology; mathematical competence; didactic resource; rural area. 


\section{INTRODUCCIÓN}

La matemática es una creación de la mente humana y su enseñanza ha de consistir en auténticos procesos de descubrimiento por parte del estudiante; pues las matemáticas no se aprenden, sino que se hacen (Sánchez, 1998). En la matemática prevalece el método sobre el contenido, de ahí la importancia de basar su enseñanza en los procesos de pensamiento matemático subyacentes a la resolución de problemas, más que a la simple transferencia de contenidos. Pues, los conceptos e ideas matemáticas se sustenta en su consideración a partir de las relaciones con los fenómenos para los que fueron creados y extendidos al proceso de aprendizaje, para mostrar al profesor los lugares por donde el aprendiz debe caminar durante su aprendizaje (Freudenthal, 2002). Este principio invita a realizar la vinculación de conceptos matemáticos con el mundo natural, cultural, social y científico; que ayuda a reflexionar sobre situaciones y contextos que facilitan la realización de actividades matemáticas adaptadas a la realidad.

Los espacios de aprendizaje no deben ser espacios inertes, por el contrario deben permitir la creación de escenarios dinámicos que promuevan el aprendizaje (Guitert y Pérez-Mateo, 2013), es decir, donde todos los estudiantes puedan aportar desde su experiencia y habilidades, en la dinámica de construcción de nuevos conocimientos; a través de una acción planificada y organizada. Donde, los objetivos de enseñanza que se plantea deben situarse en lo manipulativo y concreto y conducir al estudiante hasta lo simbólico y abstracto. Siendo fundamental, en el proceso de enseñanza-aprendizaje, la introducción de conceptos y procedimientos básicos para su ulterior aplicación, y la utilización de recursos de comprensión y exploración de la realidad contextual, que permitan la eficiencia en la resolución de operaciones algorítmicas, la realización de cálculos mentales, el uso correcto del ordenador, la estimación de medidas, cálculos, operaciones, gráficas, etc.

Los contenidos del área de matemática en la educación secundaria tiene como finalidad desarrollar las formas de actuar y pensar matemáticamente en diversas situaciones que permitan al estudiante interpretar e intervenir en la realidad a partir de la intuición, planteando supuestos, haciendo inferencias, deducciones, argumentaciones, demostraciones, formas de comunicar y otras habilidades, así como el desarrollo de métodos y actitudes útiles para ordenar, cuantificar, medir hechos y fenómenos de la realidad e intervenir conscientemente sobre ella (MINEDU, 2015, p.10). En ese sentido, la matemática escapa de ser ciencia de números y formas del espacio, para convertirse en una manera de pensar, acercarse a ella en la visión de un pensamiento organizado, formalizado y abstracto, capaz de recoger elementos y relaciones de la realidad; reconociendo como un proceso complejo y dinámico resultante de la interacción de varios factores (cognitivos, socioculturales, afectivos, entre otros), el cual promueve en los estudiantes formas de actuar y construir ideas matemáticas a partir de diversos contextos (Cantoral, 2013). Según esta afirmación, pensar matemáticamente implica entender que en el estudio de la matemática es fundamental la aproximación a todas las formas posibles de razonar, formular hipótesis, demostrar, construir, organizar, comunicar y resolver problemas matemáticos que provienen de un contexto cotidiano, social, laboral o científico; haciendo uso racional y pertinente de los recursos existentes.

En la educación básica, la tarea del docente de matemática consiste en buscar formas de mantener al estudiante motivado, interesado en la clase y en los contenidos, mostrarle lo fascinante e importante que son las matemáticas; apoyándose en estrategias de enseñanza eclécticas, en el trabajo activo y colaborativo, en comunidades de aprendizaje, en herramientas lúdicas y en el uso de tecnologías (Farías y Pérez, 2010). Teniendo en cuenta que las personas filtran el entorno a través de sus sentidos, sobre todo de aquello que le interesa y motiva, para el logro de un aprendizaje significativo en el área de matemática, por parte de los estudiantes, el profesor no solo debe compartir conocimientos, sino también disfrutar la construcción de conocimientos matemáticos orientados a la resolución de problemas de la realidad que contribuyan al desarrollo de su pensamiento matemático. Para el cual, es preciso el rediseño de estrategias didácticas sustentado en el uso pertinente de medios y recursos de enseñanza-aprendizaje.

Actualmente, en la educación secundaria del Perú, por un lado, se viene propiciando el uso de las TIC en el proceso didáctico, en forma aislada; la misma que tiene un pobre o nulo impacto en la calidad educativa que presentan algunas experiencias de introducción de TIC en la educación, basado en un proceso mecánico de "importación" de tecnologías, enfocándose únicamente en la provisión de hardware y software a las instituciones educativas, sin establecer objetivos pedagógicos claros, quedando marginado el uso de las TIC del proceso educativo (UNESCO, 2013). Bajo esta tradición la implementación de intervenciones y programas vinculados a la aplicación de TIC se caracteriza por presentar un alto grado de discontinuidad así como una debilidad notoria en sus procesos de planificación y gestión (Balarin, 2013). Por otro lado, se trata de revalorar la tecnología nativa con fines educativos; a través del uso recursos etnológicos, sustentado en la interacción directa de los estudiantes con el entorno social, cultural y físico, repercute en un proceso educativo con equidad y pertinencia, conduciendo al estudiante al desarrollo de su pensamiento numérico, algebraico y geométrico que fortalece el desarrollo de sus competencias matemáticas (Vilchez, 2018). 
Al realizar un proceso de deconstrucción de la realidad educativa en la zona rural en los cinco últimos años se perciben cambios importantes en las condiciones de vida y manifestaciones culturales de los jóvenes. En lo referente al proceso de enseñanza aprendizaje en general y, de la matemática en particular, se vienen experimentando acciones educativas sin tener en cuenta la realidad compleja de la educación en las zonas rurales, dejando a expensas el uso de las tecnologías con fines educativos. Esta afirmación es corroborada con los resultados de la encuesta aplicada a 22 profesores de matemática de zona rural (a nivel distrital a inicios del año escolar 2017) sobre el uso de tecnología digital con fines pedagógicos, solo 5 de ellos respondieron en forma afirmativa (utilizan proyector multimedia y realizan actividades con el Excel, pero no propician actividades interactivas); respecto al uso de los recursos étnicos como medio de enseñanzaaprendizaje de la matemática, 7 de los profesores respondieron que desarrollan ejercicios contextualizados en sus clases; y 2 de ellos manifiestan que usan los dos recursos. Por otro lado, en una prueba previa tomada a los estudiantes del tercer grado, 100\% obtuvieron notas muy bajas (inferior a 12 en la escala de calificación de 0 a 20), mostrando un nivel de aprendizaje deficiente.

Esta indagación, permitió identificar el uso limitado e inapropiado de recursos étnicos y digitales con fines educativos, tanto por el docente como por los estudiantes, la misma que se expresa en el deficiente desarrollo de los contenidos y objetivos de aprendizaje programados en el área de matemática. Esta dificultad, ha posibilitado implementar una estrategia del proceso de enseñanza-aprendizaje de la matemática, haciendo uso de los recursos del entorno y recursos digitales, para que el estudiante pueda construir conocimientos significativos de forma autónoma, desarrollando sus propios procesos de aprendizaje, que le permiten la construcción de su saber matemático, en la práctica de su vida cotidiana, mediante conceptos, ideas, procedimientos y actitudes; a través de los recursos del contexto y digitales como medios eficaces para potenciar sus competencias matemáticas (Pérez, 2011).

La influencia del contexto para el aprendizaje y desarrollo de las competencias matemáticas en los estudiantes del tercer grado de secundaria es fundamental; aquí el rol del docente es facilitar a los estudiantes a estructurar sus conceptos, expresar sus ideas, describir fenómenos e interpretarlo, crear problemas contextualizados al entorno y comprenderlo en forma integral; identificar los datos, traducirlo al lenguaje algebraico, para luego comunicar en forma gráfica haciendo uso de una herramienta tecnológica, mostrando actitudes y valores positivos hacia el aprendizaje de la matemática en forma interactiva y colaborativa, desarrollando su pensamiento creativo para la resolución de problemas (Ramón, 2018). Para este propósito, el uso de recursos del entorno y los digitales coadyuvan en todo proceso didáctico, porque la conjunción de ellos como recurso de aprendizaje motiva al estudiante haciéndolo un sujeto con vocación de superación y deseo de aprender la matemática desde las situaciones de la vida diaria (cultural, social y físico), mediante la resolución de problemas matemáticos provenientes del contexto con el uso de recursos digitales para la realización de cálculos numéricos y representaciones gráficas.

Desde la perspectiva docente, para la realización de una enseñanza eficaz de las matemáticas se requiere comprender lo que los estudiantes conocen y necesitan aprender y, en consecuencia, les desafía y apoya para aprender bien los nuevos conocimientos (NTCM, 2000); por ello, el estudio estuvo enfocado a implementar la inserción de tecnología mixta (nativa y digital) en el proceso didáctico y analizar la convergencia de estos dos recursos en el proceso de enseñanza y aprendizaje de los tópicos de matemática. Desde la perspectiva del estudiante, es imprescindible el cultivo de la intuición en general, la manipulación operativa del espacio y de los mismos símbolos que propicien un aprendizaje contextualizado, intencional, reflexivo, consciente y autorregulado de la matemática, orientado al logro de aprendizajes significativos. Para lograrlo, "Los estudiantes deben aprender las matemáticas con comprensión, construyendo activamente nuevos conocimientos a partir de la experiencia y los conocimientos previos". Este proceso es factible y se torna más rico con el uso de recursos étnicos y digitales, orientados a motivarlos y desarrollar sus habilidades a partir de sus estilos, los modos y formas en que aprenden a través de actividades colaborativas para un aprendizaje eficiente de los dominios de la matemática en la educación secundaria; con estudiantes activos y participativos en la construcción de sus aprendizajes y el desarrollo de su competencia matemática.

En base a la descripción y análisis de los párrafos precedentes, el estudio estuvo referido al uso de los recursos étnicos y digitales en el aprendizaje y desarrollo de competencias matemáticas en estudiantes de zonas rurales del Perú, busca responder a la pregunta: ¿Cuál es el nivel de influencia del uso de la tecnología étnica y tecnología digital, como medio y recurso didáctico, en el proceso enseñanza-aprendizaje y en el desarrollo de competencias matemáticas en los distintos dominios curriculares en los estudiantes del tercer grado de secundaria de la zona rural? Y, se tuvo como objetivo, describir la influencia del uso de la tecnología étnica y digital como recursos didácticos complementarios para el logro de aprendizajes significativos y el desarrollo de competencias matemáticas en los estudiantes del tercer grado de secundaria de la zona rural, expresada en el aprendizaje de conceptos, procedimientos y actitudes en la resolución de problemas, en los dominios de la matemática escolar. 


\section{MARCO TEÓRICO}

El proceso investigativo tuvo como referente conceptos referidos al enfoque de competencias, competencias educativas y matemáticas, los dominios de la matemática la tecnología étnica y tecnología digital; las mismas que guiaron el proceso investigativo.

\section{Enfoque de competencias}

Las competencias son procesos generales contextualizados, referidos al desempeño de la persona dentro de una determinada área del desarrollo humano, con orientación hacia la idoneidad en la realización de actividades y resolución de situaciones problemáticas que surgen en el contexto, a partir de las capacidades generales útiles para aprender en la vida diaria y en los procesos de aprendizajes cognoscitivos, emocionales y sociales; las mismas que se apoyan en los indicadores de logro como una manera de ir estableciendo su formación en etapas orientados a la integración de conocimientos, procesos cognitivos, destrezas, habilidades, valores y actitudes en el desempeño; así como también considera requerimientos de las disciplinas, profesiones y contextos (Tobón et al, 2010). Identificándose que las competencias genéricas son comunes a todas las áreas; mientras, las específicas que dan identidad a cada área o dominio, y en cualquiera de los casos, implican perfiles de desempeño.

Para Tobón (2015), las competencias son procesos complejos que las personas ponen en acción-actuacióncreación, para resolver problemas y realizar actividades (de la vida cotidiana y del contexto laboral profesional), aportando a la construcción y transformación de la realidad, para lo cual integran el saber ser (automotivación, iniciativa y trabajo colaborativo con otros), el saber conocer (observar, explicar, comprender y analizar) y el saber hacer (desempeño basado en procedimientos y estrategias), teniendo en cuenta los requerimientos específicos del entorno, las necesidades personales y los procesos de incertidumbre, con autonomía intelectual, conciencia crítica, creatividad y espíritu de reto, asumiendo las consecuencias de los actos y buscando el bienestar humano. Las competencias, en tal perspectiva, están constituidas por procesos subyacentes (cognitivo-afectivos) así como también por procesos de afrontar la resolución de problemas poniendo a flote el raciocinio lógico y la creatividad.

\section{Las competencias en el ámbito educativo}

Una competencia va más allá de conocimientos y habilidades para afrontar demandas complejas en un contexto particular, ya que la existencia de un saber es complejo y resulta de la integración, movilización y adecuación de capacidades, conocimientos, actitudes, valores, utilizados eficazmente en situaciones reales por los individuos, de tal forma que toda competencia incluye un "saber", un "saber hacer" y un "saber ser", en contextos y situaciones concretas en función de propósitos deseados (Pérez, 2011).

Entre las ventajas y aportes de la Educación basada en Competencias destacan: la valorización, a nivel del mundo laboral, de las personas y su experiencia; la relevancia en el vínculo que se propicia entre teoría y práctica, mundo disciplinario y mundo laboral, la flexibilización de los diseños curriculares centrados en asignaturas y avanzando en propuestas medulares relativas a necesidades formativas derivadas de la práctica; convergencia del quehacer docente de las unidades académicas, colaborando en la construcción de un proyecto pedagógico común; permitiendo la innovación en los sistemas de evaluación del aprendizaje, ampliando el espectro de los instrumentos de acuerdo a la realidad, dando prioridad al estudiante (Rey, 1998).

Las competencias se basan en indicadores de desempeño y estos corresponden a los indicadores de logro y las metas por alcanzar en procesos pedagógicos en su integralidad, mientras que los estándares se refieren a metas específicas por lograr durante las fases de dicho proceso. El desarrollo de competencias educativas a nivel institucional, curricular y profesor-estudiante. A nivel Institucional, referidos a la implementación de métodos de enseñanza, aprendizaje y evaluación, basados en la implementación de programas y capacitación de los profesores, y al proceso de enseñanza-aprendizaje sobre la base de competencias establecidas. A nivel curricular se explicitan las competencias específicas; las asignaturas de estudios; los objetivos a lograr; las modalidades de conducción y evaluación de los contenidos para verificar el logro de competencias en los estudiantes. La Interacción profesor-estudiante, referido a: métodos de enseñanza, aprendizaje y evaluación por competencias, que se lleva a cabo en el salón de clases, laboratorio, talleres y actividades extracurriculares.

\section{Competencias matemáticas}

Según (NCTM, 2000), la actividad matemática aparece como una serie de procesos que se articulan en su estudio, cuando los estudiantes interaccionan con situaciones y problemas, bajo la dirección y el apoyo del profesor, a través de resolución de problemas, que implica exploración de las posibles soluciones, 
modelización de la realidad; desarrollo de estrategias y aplicación de técnicas; representación, sustentado en el uso de recursos verbales, simbólicos y gráficos, traducción y conversión entre los mismos; comunicación, diálogo y discusión estudiante-estudiante y profesor-estudiante; justificación, dados a través de distintos tipos de argumentación que pueden ser deductivas, inductivas, constructivas, etc.; conexión, referido al establecimiento de relaciones entre distintos objetos matemáticos. Estos procesos se articulan a lo largo de la enseñanza de los contenidos matemáticos a través de situaciones didácticas en el aula.

La competencia matemática de los escolares se expresa en "la capacidad individual para identificar y comprender el papel que desempeñan las matemáticas en el mundo, emitir juicios bien fundados, utilizar las matemáticas y comprometerse con ellas y satisfacer las necesidades de la vida personal como ciudadano constructivo, comprometido y reflexivo" (OECD, 2003). Por otra parte, "la competencia considerada en el ámbito de las matemáticas debe explicar y predecir el conjunto, potencialmente infinito, de las actuaciones del sujeto. Sin embargo, también se puede hablar de la competencia en un dominio más o menos concreto de las matemáticas, de manera que el modelo de competencia tiene que describir la conducta del sujeto ideal en ese dominio y, así, explicar y predecir su conjunto de actuaciones posibles en ese dominio" (Puig, 2008).

De otro lado, pensar matemáticamente consiste en el conjunto de actividades mentales u operaciones intelectuales que llevan al estudiante a entender y dotar de significado a lo que le rodea, resolver un problema usando conceptos matemáticos, tomar una decisión o llegar a una conclusión, en los que están involucrados procesos como la abstracción, justificación, visualización, estimación, entre otros (Cantoral et al, 2005). Constituyéndose la matemática en un medio para describir, comprender e interpretar los fenómenos naturales y sociales que han motivado el desarrollo de determinados procedimientos y conceptos matemáticos propios de cada situación o dominio, usándola para describir, comprender y actuar en diversos contextos; mediante el planteo y resolución de problemas.

\section{Dominios en el área de la matemática escolar}

Según la (UNESCO, 2016) en la educación secundaria son prioritarios que los estudiantes desarrollen cinco competencias o dominios que son: el numérico, geométrico, medición, estadístico y de variación; los mismos que se logran a través del aprendizaje de conceptos, desarrollo de procedimientos y de actitudes.

Dominio numérico: referidos a sistemas de numeración, operaciones y reglas de uso de tecnología. Sustentado en procedimientos como estrategias para contar, explicación oral del proceso seguido en la realización de cálculos y en la resolución de problemas numéricos, estimación de los resultados de un cálculo, elaboración de estrategias personales de cálculo mental y uso de la tecnología para realizar operaciones numéricas. Desarrollando actitudes de la curiosidad por indagar y explorar sobre el significado de códigos numéricos y regularidades que aparecen en conjuntos numéricos, sensibilidad e interés por las informaciones y mensajes de naturaleza numérica, apreciando la utilidad de los números en la vida cotidiana, confianza propia en sus capacidades y las estrategias de realizar cálculos mentales, presentación ordenada y clara de los cálculos y de sus resultados.

Dominio geométrico: sustentado en el estudio de distancias, ángulos y giros, sistema de coordenadas cartesianas; relación entre elementos geométricos: paralelismo y perpendicularidad; representación del espacio: planos, mapas y maquetas; formas planas y espaciales; regularidades y simetrías. Todo ello, mediante procedimientos de descripción de la situación y posición de un objeto en el espacio con relación a un sistema de referencia; interpretación y descripción de croquis, planos, maquetas y mapas; comparación y clasificación de figuras y cuerpos geométricos utilizando diversos criterios; formación de figuras planas y cuerpos geométricos a partir de otras por composición o descomposición. Orientado al desarrollo de actitudes como la valoración de la utilidad de los sistemas de referencia y de la representación espacial en actividades cotidianas; sensibilidad y gusto por la elaboración y la presentación cuidadosa de las construcciones geométricas; precisión en los cálculos de los elementos geométricos.

El dominio de la medición: referido a la necesidad y funciones de la medición, unidades no convencionales de medición, unidades de medida del sistema internacional, unidades de medida del tiempo. A través de procedimientos de mediciones con unidades convencionales y no convencionales, elaboración y estimación de estrategias personales para llevar a cabo estimaciones de medidas en situaciones cotidianas, toma de decisiones sobre unidades de medidas más adecuadas de acuerdo al objetivo de la medición, expresión verbal del proceso seguido en el proceso de medición. Para el desarrollo de la valoración de la importancia de las mediciones y estimaciones en la vida cotidiana, gusto por la precisión apropiada en la realización de mediciones, tendencia a expresar los resultados numéricos de las mediciones manifestando las unidades de medidas utilizadas. 
Dominio estadístico: capacidad de construir tablas de datos, tipos de gráficos estadísticos, carácter aleatorio de algunas experiencias. A través de procedimiento de exploración sistemática e interpretación de datos de una tabla estadística, recolección y registro de datos sobre objetos, fenómenos y situaciones diversas utilizando técnicas elementales de encuesta, observación y medición; elaboración de gráficas estadísticas; realización de experimentos, espacio muestral, eventos y probabilidad de un evento. Para propiciar actitud crítica sobre las informaciones transmitidas mediante cuadros y gráficos, valoración de la expresividad de las gráficas estadísticas observadas y elaboradas. Interpretación de situaciones, selección, recolección, organización e interpretación de información.

Dominio de variación: orientado a identificar regularidades y patrones numéricos y geométricos en representaciones diversas. Identificación de variables y la interpretación de situaciones en las que se distinguen las mismas. Descripción de fenómenos de cambio y dependencia, que considera la resolución de problemas y la valoración de la pertinencia del proceso seguido. Noción de función, uso de conceptos y procedimientos asociados a la variación directa, a la proporcionalidad y a la variación inversa en contextos aritméticos y geométricos en la resolución de problemas.

\section{Los recursos étnicos en la enseñanza de la matemática}

Una actividad matemática es la exploración de ciertas estructuras de la realidad, entendida esta en sentido amplio, como realidad física y mental; y se enfrenta con cierto tipo de estructuras que se prestan a unos modos peculiares de tratamiento que incluyen: una simbolización adecuada, que permite presentar eficazmente, desde un punto de vista operativo, las entidades que maneja; una manipulación racional rigurosa, que contemple al ascenso de aquellos que se adhieren a las convenciones iniciales de partida; un dominio efectivo de la realidad a la que se dirige, primero racional, del modelo mental que se construye, de la realidad exterior modelada (De Guzmán y Gil, 1993).

Para generar materiales contextualizados para el entorno escolar de las comunidades. Este proceso requiere indagaciones sobre la naturaleza antropológica y etnológica, donde es posible mostrar diversos hallazgos etnográficos considerados como aportes a la dimensión histórica de los conocimientos matemáticos como un producto del quehacer humano. Su proceso de construcción está sustentado en abstracciones sucesivas surgidas desde la realidad (D'Ambrosio, 2005). Desde esta perspectiva, el proceso de enseñanza-aprendizaje de la matemática establece conexiones entre cultura, matemática, historia, geografía, antropología y otras ciencias sociales; a partir del conocimiento matemático de los pueblos que se expresan a través de los cálculos y mediciones que se realizan a entes naturales y artificiales (caminos, carrizos, árboles, cerámica, arte rupestre, rocas, sembríos, etc.).

Las actividades de aprendizaje deben estar orientados a generar espacios de construcción colectiva del conocimiento matemático a partir de su cultura, creencias, cosmovisión, constructos personales (Fuentes, 2014). Por ello, es fundamental que toda actividad matemática esté relacionado con actividades diarias de los estudiantes, pues un problema matemático afecta inmediatamente al individuo y al modo en que este percibe el problema conectado a un entorno laboral o de trabajo, a través de la construcción (replanteos, parcelación,...), trazado de ángulos rectos, medidas de distancias y pendientes,... que son recursos innatos de la comunidad, en la cual los estudiantes observan determinados aspectos de su entorno o que aparezcan en los medios de comunicación.

\section{Tecnología digital en la enseñanza de la matemática}

La tecnología digital se utiliza en las modalidades: e-learning, que permite gran flexibilidad respecto de los espacios y los tiempos, pero dependiente de la necesidad física de un ordenador y una conexión a Internet; y el m-learning, relacionado con el aprendizaje ubicuo ligado a la movilidad y al contexto de aprendizaje del estudiante a través de celulares; ambas fundamentales como recurso de enseñanza-aprendizaje. El estudiante tiene escasa conciencia que el móvil puede ser utilizado con fines educativos, pues utilizan solo como medio comunicacional y lúdico; también el profesorado desconoce la potencialidad educativa de estos dispositivos, consideran como elemento de intromisión en el aula. Al respecto (Brazuelo y Cacheiro, 2015) consideran que la amplia proliferación del teléfono móvil constituye un elemento integrante de la vida cotidiana e imprescindible en los estudiantes, y suponen una oportunidad para extender su uso con fines educativos, ya que los estudiantes están empoderados de la tecnología digital.

El empoderamiento digital se sustenta en el uso cotidiano del ordenador y los Smartphone; pero, estos recursos son utilizados, por la mayoría de docentes y estudiantes, con fines lúdicos y comunicacionales; lo que se trata es de que estos recursos se inserten como medio y recurso para realizar tareas, resolver problemas, comunicar, gestionar la información; colaborar, crear y compartir contenidos y construir el conocimiento de manera eficaz, eficiente, adecuadamente; de manera crítica, creativa, autónoma, con 
flexibilidad; de manera ética, reflexiva para el trabajo; con mayores posibilidades de comunicación e interacción desde cualquier lugar y en cualquier momento. La incorporación de la tecnología digital en el proceso de enseñanza-aprendizaje de los estudiantes de zona rural, permitieron la realización de tareas interactivas mediante el uso de software libre (Geup y Geogebra) y uso de la página interactiva (ThatQuiz), para realizar trabajos colaborativos el Google Drive, y entrega de tareas el Facebook y el WhatsApp.

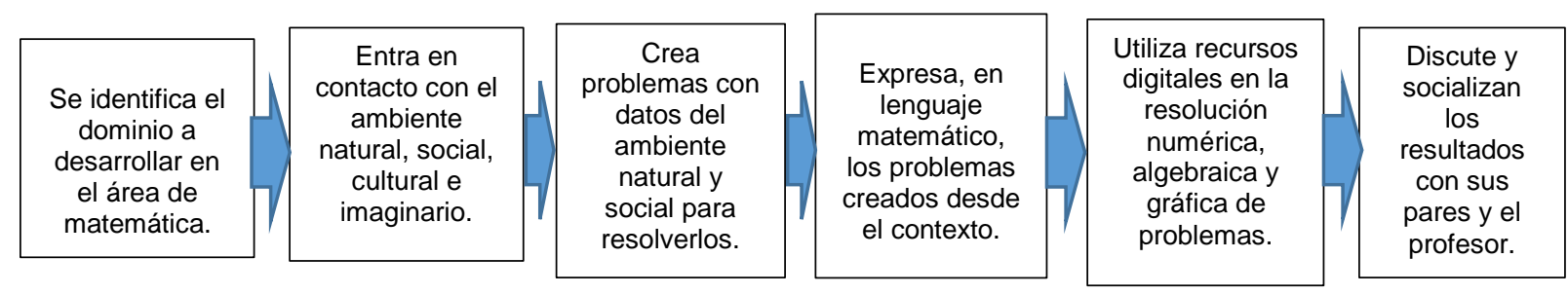

Fig. 1: Proceso de aprendizaje seguido con uso de recurso étnico y digital en el estudio de la matemática.

Una buena enseñanza-aprendizaje de la matemática exige buenas condiciones ambientales y didácticas en las instituciones educativas. Además necesita paciencia, tiempo y recursos; pero fundamentalmente contar con profesores conocedores del entorno socio-físico-cultural y de las tecnologías emergentes, que ayude a los estudiantes en la elaboración de los conceptos; originar un entorno propicio para el aprendizaje, basado en contextos cercanos a la experiencia cotidiana los que tienen sentido para el estudiante, fortaleciendo la predisposición positiva hacia el aprendizaje, inmerso en un contexto étnico y digital, afianza en el estudiante su pensamiento y cultura matemática sustentado en los recursos de su cultura ancestral en armonía con los recursos y medios de la tecnología digital. Donde los estudiantes manifiesten motivación y participación activa en la construcción de su conocimiento matemático, aspecto fundamental para lograr aprendizajes significativos e útiles en la aplicación de esta ciencia a la solución de problemas (Morales-Maure et al, 2018).

\section{METODOLOGÍA}

La estrategia metodológica consistió en la realización de una investigación mixta con preponderancia cualitativa. La población de estudio lo conforman adolescentes cuyas edades fluctúan entre 13 y 15 años, provenientes de hogares dedicados a la agricultura y ganadería, que están cursando el tercer grado de educación secundaria en 12 instituciones educativas rurales de Huánuco; la muestra elegida en forma intencional lo conforman 15 estudiantes de la comunidad de Huancanyacu, la misma que es representativa considerando las características homogéneas de los estudiantes de zonas rurales de la región Huánuco, ubicada al nororiente del Perú.

Para iniciar el proceso investigativo se realiza un estudio exploratorio sobre el uso de la tecnología étnica y digital con fines educativos en el contexto de la educación rural y analizar la posibilidad de su inserción como recurso didáctico. En concordancia con los hallazgos obtenidos en la prueba previa y resultados de la encuesta a los profesores, se opta por un diseño de investigación descriptivo y de ejecución concurrente, en la modalidad de investigación-acción. La investigación acción tuvo las fases: observar (construir un bosquejo del problema y recolectar datos), pensar (analizar e interpretar) y actuar (resolver problemas e implementar mejoras), las cuales se dan de manera cíclica, una y otra vez, hasta que el problema es resuelto, el cambio se logra o la mejora se introduce satisfactoriamente (Stringer, 2014). En el proceso de ejecución concurrente, los datos cuantitativos y cualitativos se recolectan y analizan casi al mismo tiempo, otorgándose a las dos formas de datos la misma importancia, luego, los resultados de las etapas cuantitativa y cualitativa son integradas para el proceso de interpretación (Hernández et al, 2014).

El trabajo de campo se lleva a cabo en tres etapas: diagnóstica, de intervención y de evaluación. En la etapa diagnóstica, después de la prueba previa, se identificaron las necesidades de aprendizaje, algunos recursos étnicos y digitales utilizables, los temas a tratar y las competencias a lograr en el contexto en donde se desarrolla el proceso de enseñanza-aprendizaje, y se lleva a cabo en la primera semana del segundo bimestre del año escolar. En la etapa de intervención se lleva a cabo la estrategia idónea durante seis semanas de clase, dos sesiones por semana, abordándose un dominio en cada semana. Y, en la etapa de evaluación se realiza una reflexión multidireccional de las actividades cumplidas y de los resultados obtenidos, utilizando los instrumentos de recolección de datos e información diseñado por los investigadores.

Para la recolección de datos se utilizaron como instrumentos: La lista de cotejo, instrumento de medición observacional de los aprendizajes esperados de los estudiantes de manera cualitativa, para valorar el desarrollo de sus competencias matemáticas mediado por recursos étnicos y digitales. La rúbrica elaborada en relación con los objetivos de aprendizaje para la evaluación del uso conceptos, procedimentales y habilidades en la resolución de problemas matemáticos. Prueba cognitiva, consistió en una cuestionario de cinco problemas por dominio, con una calificación vigesimal ( 0 a 20), los que se administraron durante 30 
minutos cada uno; y el cuestionario de satisfacción, orientado a medir el nivel de conformidad que tienen los estudiantes respecto a la estrategia didáctica implementa.

Para la validación de los hallazgos, desde la perspectiva cualitativa se analiza los juicios emitidos por los estudiantes sobre la pertinencia del uso de recursos del contexto y digitales para el estudio y desarrollo de sus competencias matemáticas; mientras, desde la óptica cuantitativa se elaboran cuadros estadísticos y se calculan medidas estadísticas descriptivas. En la presentación y análisis de datos se empleó el software estadístico Minitab, el mismo que facilitó la interpretación de los datos y la generación de información verídica de la experiencia pedagógica.

\section{RESULTADOS}

En base en los datos obtenidos podemos dar respuesta a la pregunta de investigación ¿Cuál es el nivel de influencia del uso de la tecnología étnica y tecnología digital, como medio y recurso didáctico, en el proceso enseñanza-aprendizaje y en el desarrollo de competencias matemáticas en los distintos dominios curriculares en los estudiantes del tercer grado de secundaria de la zona rural? La respuesta se sustenta a través de resultados obtenidos en lista de cotejo, rúbrica, prueba y cuestionario de satisfacción.

En la lista de cotejo referido al logro de competencias matemáticas en los cinco dominios que corresponden al estudio de la matemática escolar, a través de las actividades realizadas en el proceso de enseñanzaaprendizaje de la matemática, utilizando la tecnología étnica y digital como medio y recurso didácticos. Teniendo como indicadores: nivel de motivación para el estudio, habilidad para resolver problemas, elaboración y recreación de conceptos, el modelamiento y el proceso de comunicación matemática: la mayoría, más $62 \%$, de los estudiantes desarrollaron en forma significativa sus competencias matemáticas, en los cinco dominios, a través de un estudio sistemático de los contenidos: con inicio en la observación y manipulación de recursos étnicos, seguido de un proceso de matematización, conceptualización y modelación, para luego realizar las operaciones algebraicas y representaciones gráficas haciendo uso de los recursos digitales (figura 2).

Competencia Matemática

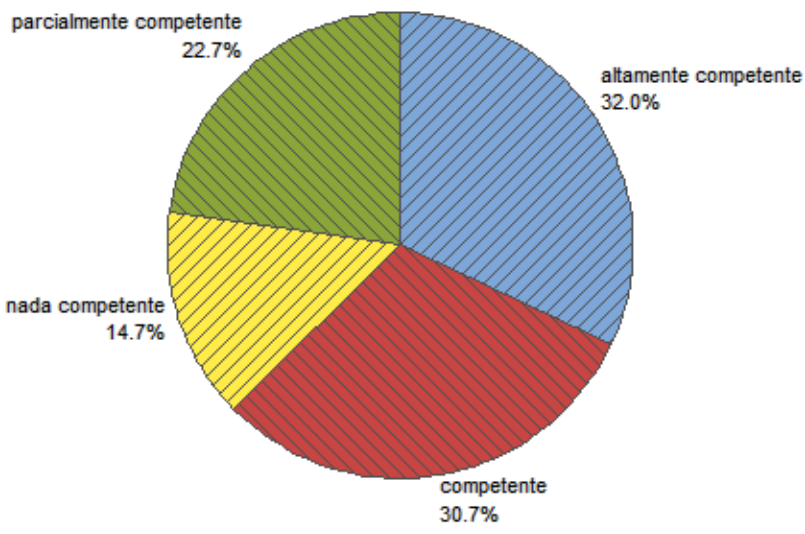

Fig. 2: Nivel de competencias matemáticas desarrolladas en los cinco dominios.

Los resultados de la aplicación de la rúbrica de evaluación del aprendizaje logrado de los contenidos matemáticos a nivel conceptual, procedimental y actitudinal, teniendo en cuenta los aspectos considerados en los cinco dominios. Los resultados obtenidos se detallan en la tabla 1, donde más del $64 \%$ de los participantes lograron un nivel de aprendizaje excelente o bueno; el 28,89\% lograron un aprendizaje regular; y, solo el $6,67 \%$ de los estudiantes tuvieron un aprendizaje deficiente, la misma podemos deducir que con el uso de la tecnología digital y étnica, el aprendizaje logrado fue lo esperado.

Tabla 1: Resultados de evaluación conceptual, procedimental y actitudinal.

\begin{tabular}{|c|c|c|c|c|}
\hline \multirow{2}{*}{$\begin{array}{c}\text { Aprendizaje } \\
\text { logrado }\end{array}$} & \multicolumn{4}{|c|}{ Niveles de aprendizaje } \\
\cline { 2 - 5 } & Conceptual & Procedimental & Actitudinal & Total \\
\hline Excelente & $4(8,89 \%)$ & $4(8,89 \%)$ & $3(6,67 \%)$ & $11(24,44 \%)$ \\
\hline Bueno & $5(11,11 \%)$ & $7(15,56 \%)$ & $6(13,33 \%)$ & $18(40,00 \%)$ \\
\hline Regular & $5(11,11 \%)$ & $3(6,67 \%)$ & $5(11,11 \%)$ & $13(28,89 \%)$ \\
\hline Deficiente & $1(2,22 \%)$ & $1(2,22 \%)$ & $1(2,22 \%)$ & $03(06,67 \%)$ \\
\hline Total & $15(33,33 \%)$ & $15(33,33 \%)$ & $15(33,33 \%)$ & $45(100,00 \%)$ \\
\hline
\end{tabular}


Los calificativos registrados en la prueba cognitiva administrada a los sujetos de la investigación con ítems correspondientes a los cinco dominios, se realiza al final del primer semestre del año 2017, con una escala de calificación vigesimal (de 0 a 20), clasificado en cuatro niveles (intervalos). Más del $68 \%$ de los participantes lograron un nivel de aprendizaje destacado-satisfactorio; mientras que $20,00 \%$ se le atribuyó el nivel suficiente, y sólo el $12,00 \%$ se ubicó en nivel de logro insuficiente. En resumen se puede afirmar que la mayoría de los estudiantes tuvieron calificativos mayores a la nota 14, ello significa que el uso de recursos étnicos y digitales favorecen un aprendizaje eficiente de la matemática, en los cinco dominios del área de matemática, considerados para la educación secundaria (figura 3).

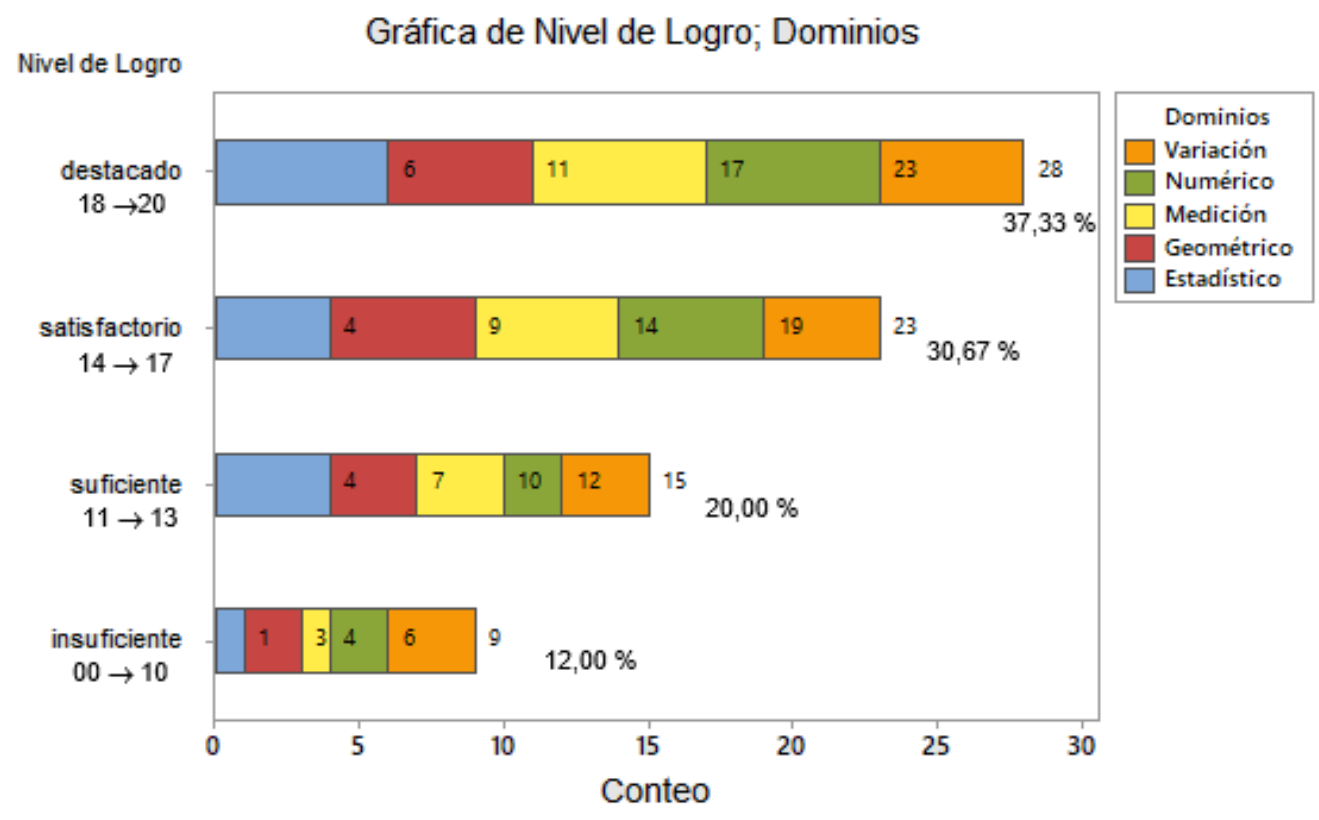

Fig. 3: Resultados de evaluación de los estudiantes según dominio y nivel de logro.

Respecto al nivel de satisfacción referidos a la enseñanza recibida del docente, el aprendizaje logrado en los cinco dominios, sobre las actividades realizadas en el proceso de enseñanza y aprendizaje, y referido al uso de la recursos étnicos y digitales como medio y recurso para el aprendizaje de la matemática. Como se muestra en la tabla 2, más del $70 \%$ de los estudiantes manifiestan estar satisfechos con la forma de llevar a cabo la enseñanza y aprendizaje de la matemática, mientras los que se muestran insatisfechos no sobrepasan el 3,00\%. Es decir, en los tres niveles de aprendizaje, los recursos tecnológicos étnicos y digitales utilizados en proceso didáctico, influyeron significativamente en el nivel de satisfacción de los estudiantes.

Tabla 2: Nivel de satisfacción de los estudiantes con la estrategia didáctica implementada.

\begin{tabular}{|c|c|c|c|c|c|c|}
\hline \multirow{2}{*}{$\begin{array}{c}\text { Nivel de } \\
\text { satisfacción }\end{array}$} & \multicolumn{6}{|c|}{ Elementos del proceso enseñanza-aprendizaje } \\
\cline { 2 - 7 } & $\begin{array}{c}\text { Actividades } \\
\text { desarrolladas }\end{array}$ & $\begin{array}{c}\text { Aprendizaje } \\
\text { logrado }\end{array}$ & $\begin{array}{c}\text { Enseñanza } \\
\text { recibida }\end{array}$ & $\begin{array}{c}\text { Tecnología } \\
\text { étnica }\end{array}$ & $\begin{array}{c}\text { Tecnología } \\
\text { digital }\end{array}$ & Total \\
\hline Muy satisfecho & 4 & 5 & 4 & 4 & 5 & 22 \\
& $(5,33 \%)$ & $(6,67 \%)$ & $(5,33 \%)$ & $(5,33 \%)$ & $(6,67 \%)$ & $(29,33 \%)$ \\
\hline Satisfecho & 7 & 6 & 7 & 6 & 5 & 31 \\
$(9,33 \%)$ & $(8,00 \%)$ & $(9,33 \%)$ & $(8,00 \%)$ & $(6,67 \%)$ & $(41,33 \%)$ \\
\hline $\begin{array}{c}\text { Parcialmente } \\
\text { satisfecho }\end{array}$ & $4(5,33 \%)$ & 4 & 3 & 4 & 5 & 20 \\
\hline $\begin{array}{c}\text { Nada } \\
\text { satisfecho }\end{array}$ & 0 & $(5,33 \%)$ & $(4,00 \%)$ & $(5,33 \%)$ & $(6,67 \%)$ & $(26,67 \%)$ \\
\hline Total & 15 & 0 & 1 & 1 & 0 & 2 \\
& $(20,00 \%)$ & $(20,00 \%)$ & $(20,00 \%)$ & $(20,00 \%)$ & $(20,00 \%)$ & $(100,00 \%)$ \\
\hline
\end{tabular}

\section{DISCUSIÓN}

Tradicionalmente la mayoría de los profesores, creen utilizar diversas actividades, pero siempre dependen de los contenidos a través de actividades con el fin de que los alumnos resuelvan problemas y comprendan los contenidos, además de comprobar la teoría (Azcarate y Cuesta, 2005). Como alternativa a este procedimiento tradicional, para un desarrollo integral de las capacidades y competencias matemáticas en los estudiantes, 
es conveniente llevar el proceso enseñanza-aprendizaje: a) basado en un currículum en función de sus implicaciones en un contexto socioculturalmente distinto al oficial; b) dar cuenta de procesos matemáticos que realizan diversos grupos culturales con foco en el reconocimiento del saber cultural, para la enseñanza y el aprendizaje de la matemática; c) dar cuenta de la implicaciones de estos conocimientos culturales en el ámbito de la educación formal, y d) las concepciones en educación matemática de profesores y estudiantes en nexo a las líneas de análisis etnomatemático, para transparentar las distintas barreras educacionales antes y durante el proceso de enseñanza y aprendizaje de las matemáticas (Huencho, 2015). Por otro lado, es imprescindible enfatizar la formación del profesorado desde la mediación didáctica de las TIC, con la intención de fortalecer su práctica pedagógica e incidir en la cualificación del docente, asociada al desarrollo de capacidades para responder de forma ágil y oportuna a los desafíos propios de la era digital (Marín et al, 2017). La puesta en práctica de las ideas descritas permiten reforzar el aprendizaje de conceptos, procedimientos y en el desarrollo de actitudes de los estudiantes, hecho que se evidencia en el resultado obtenido desde la rúbrica de evaluación de competencias.

Los resultados obtenidos en la prueba cognitiva (más del 85\% de aprobados con notas mayores a 10), evidencian la eficacia que tienen el uso de la tecnología nativa y la tecnología moderna para la optimización del proceso de aprendizaje de la matemática; permitiendo al estudiante y docente valorar la cultura de la comunidad y la tecnología para el aprendizaje sostenido de la matemática; cambiando sus roles y responsabilidades en el proceso didáctico, donde se pone énfasis en la propia actividad, los procesos de dotar de significado a la tarea, los procesos de solución, y la propia solución a los problemas. Ratificando, que el estudio estuvo orientado a subsanar la falencia de los profesores de no utilizar la etnomatemática en el aula de clases y en los ambientes virtuales, porque no poseen una formación adecuada y no están insertos en la tendencia educativa actual; a través de actividades instructivas que faciliten la contextualización y la integración del saber académico con lo cotidiano para que esos saberes sean utilizados en la acción pedagógica de enseñanza y aprendizaje de la matemática (Rosa y Clark, 2013).

El diseño e implementación de la práctica pedagógica deben convertirse en una dinámica flexible que se oriente al desarrollo del estudiante y se encamine a resolver situaciones reales del entorno cotidiano, en el marco de promover el trabajo en equipo, colaborativo y toma de decisiones; donde la comunicación del docente se torna eficiente, cuando se reconocen como sujetos con oportunidad de aportar a través de espacios y estrategias de enseñanza-aprendizaje de la matemática (Martínez, 2016). También, la actuación del docente debe estar presidida por estos tres principios: 1) incluir a los estudiantes en decisiones sobre cómo y qué deben aprender y evaluar el aprendizaje; 2) respetar y tener en cuenta las diferencias individuales relativas al entorno, intereses, capacidades y experiencias y 3) tratar a los alumnos como co-creadores del proceso de enseñanza (Sáenz y Lebrija, 2014). Por ello, en la educación actual, independientemente de las estrategias docentes, es algo ineludible el uso de la tecnología digital, pero también rescatar la versatilidad que tienen los recursos étnicos como medio y material didáctico en los distintos dominios de la matemática, que facilitan en forma conjunta el logro de aprendizajes eficientes y el desarrollo de competencias; como fue corroborado en las respuestas al cuestionario de satisfacción, donde más del 95\% se muestran conformes con las actividades realizadas, la enseñanza recibida, el aprendizaje logrado mediado por las tecnologías.

Las tecnologías son bastante heterogéneos y cambian con el tiempo y el enfoque deseado, sirven de apoyo didáctico de los procesos de aprendizaje (Muller y Strohmeier, 2011). Por otro lado, la percepción que la tecnología no aporta a las relaciones interpersonales y a la calidad de la enseñanza, la tecnología aún no ha logrado colocarse como un elemento robusto en la educación y solo es utilizada como elemento de comunicación o de uso recreativo consistente, la primera razón de uso de internet es la comunicación y solo en la tercera posición el uso educativo (Rodríguez et al, 2017). Entonces para robustecer el uso de la tecnología en la educación es imprescindible la realización de actividades interactivas y colaborativas, tanto en el ambiente real y el ambiente digital, implementando nuevas estrategias didácticas acorde a la realidad física y la realidad tecnológica; promoviendo el uso de la tecnología étnica y la tecnología digital como medio y no como fin, tanto los docentes como los estudiantes. Poniendo énfasis que las tecnologías utilizadas involucren la posibilidad de explorar y coordinar, por parte de los estudiantes, diferentes representaciones, visualizaciones y en el análisis de datos numéricos, que sirvan como insumo para la realización de operaciones numéricas, algebraicas y construcción de representaciones gráficas. Hecho que fue se evidencia con los resultados obtenidos a través de la lista de cotejo de las actividades en clase.

Finalmente, consideramos de importancia resaltar que la investigación consistió en observar, describir y analizar las actividades matemáticas de los estudiantes del tercer grado de secundaria, así como la actuación del profesor durante el proceso, siendo esta manera de proceder de índole objetivo. Durante el proceso didáctico se prioriza el uso de recursos étnicos, como ello no fue suficiente, se complementa con actividades interactivas que conducen a procesos de razonamiento y abstracción, orientado al desarrollo del pensamiento matemático del estudiante; para luego incursionar a la tecnología digital para el procesamiento de información y comunicación matemática en los cinco dominios abordados. 


\section{CONCLUSIONES}

El proceso de enseñanza y aprendizaje de la matemática haciendo uso de los recursos étnico-digitales, donde los estudiantes interactúan en forma directa con su entorno social, cultural, físico y tecnológico, coadyuvan a la implementación de un proceso educativo con equidad y pertinencia, conduciendo al estudiante a desarrollar su pensamiento numérico, algebraico y geométrico de manera activa, a través de la manipulación de objetos concretos de su entorno y de los recursos digitales; la misma que mejora su nivel de motivación para el estudio, habilidad para resolver problemas, elaboración y recreación de conceptos, el modelamiento, la resolución de problemas y el proceso de comunicación matemática; fortaleciendo su competencia matemática, como muestran los resultados de la lista de cotejo.

El uso de la tecnología étnica y digital con fines educativos, tiene efectos muy positivos en el aprendizaje de la matemática por parte de los estudiantes de la zona rural, pues permite el logro de aprendizajes significativos, a través de la realización de actividades interactivas; práctica de trabajos colaborativos y estudio cooperativos, haciendo uso de las redes sociales y software interactivo, las mismas que repercuten en su aprendizaje conceptual, procedimental y desarrollo de actitudes favorables para el estudio y autoestudio, como se evidencia en los resultados de la rúbrica.

Los resultados obtenidos de la prueba cognitiva y el cuestionario de satisfacción, evidencian que la tecnología étnica y digital son recursos estratégicos convergentes para el desarrollo de aprendizajes significativos y satisfacción plena de los estudiantes de la zona rural. Su uso genera escenarios motivadores para el aprendizaje constructivo de la matemática, partiendo el proceso, desde los recursos manipulables del entorno, la matematización, la modelación y posterior obtención de resultados haciendo uso de recursos digitales, en los distintos dominios de la matemática, a través de actividades de aprendizaje interactiva y colaborativa, donde el conocimiento matemático se construye desde la realidad para resolver problemas de la realidad, propiciando la formación de personas integrales en una sociedad plural y tecnológica.

Finalmente es preciso manifestar que, actualmente es imprescindible el uso de los recursos étnicos y digitales en el proceso de enseñanza-aprendizaje de la matemática en la educación rural; ya que además de servir como nuevos medios para optimizar el aprendizaje, suponen la adquisición de competencias necesarias entre los estudiantes en la construcción de su aprendizaje. En este camino, los profesores de matemática deben ser los propulsores y creadores de la forma óptima de usar estos recursos de tal manera que los estudiantes se mantengan siempre motivados y sus aprendizajes sean cada vez más significativos.

\section{REFERENCIAS}

Azcarate, P. y J. Cuesta, El profesorado novel de secundaria y su práctica. Estudio de un caso en las áreas de ciencias, Enseñanza de las Ciencias: revista de investigación y experiencias didácticas, 23 (3), 393-402 (2005)

Balarin, M., Las políticas TIC en los sistemas educativos de América Latina - Caso Perú. Fondo de las Naciones Unidas para la Infancia (UNICEF) (2013)

Brazuelo, F. y M.L. Cacheiro, Study of Adaptability of MOOC Platforms to Mobile Devices, doi: 10.6018/red/47/1, REDRevista de Educación a Distancia, 47 (1) 1-13 (2015)

Cantoral, R., Teoría socioepistemológica de la matemática educativa. Estudios sobre la construcción social del conocimiento, Gedisa, México (2013)

Cantoral, R., R.M. Farfán y otros cuatro autores, Desarrollo del pensamiento matemático, Trillas, México (2005)

D’Ambrosio, U.O., Programa Etnomatemática como uma proposta de reconhecimento de outras formas culturais, doi: https://doi.org/10.14409/yu.v1i2.245, Yupana, 1(2), 63-71 (2005)

De Guzmán, M. y D. Gil, La enseñanza de las ciencias y la matemática. Tendencias e innovaciones, Popular, Madrid, España (1993)

Farias, D. y J. Pérez, Motivación en la Enseñanza de las Matemáticas y la Administración; Formación Universitaria, doi: 10.4067/S0718-50062010000600005, 3(6), 33-40 (2010)

Freudenthal, H., Didactical Phenomenology of Mathematical Structures, $2^{a}$ Ed., Kluwer Academic Publushers, New York, USA (2002)

Fuentes, C., Descolonizando la escuela: ¿Es Posible Llevar la Etnomatemática al aula?, Revista Latinoamericana de Etnomatemática, 7(2), 222-244 (2014)

Guitert, M. y M. Pérez-Mateo, La colaboración en la red: Hacia una definición de aprendizaje colaborativo en entornos virtuales, E-ISSN: 1138-9737, Teoría de la Educación. Educación y Cultura en la Sociedad de la Información (TESI), 14(1), 10-31 (2013)

Hernández, R., C. Fernández y M. Baptista, Los métodos mixtos. Metodología de la investigación, 6ª Ed., 531-557, McGraw-Hill, México (2014) 
Huencho, A., La Etnomatemática en contexto de la Educación Formal: una revisión de literatura, Conferencia interamericana de educación matemática: XIV CIAEM-IACME, Chiapas, México 15(1) 1-12 (2015)

Marín, V., A. Inciarte, H. Hernández y R. Pitre, Estrategias de las Instituciones de Educación Superior para la Integración de las Tecnología de la Información y la Comunicación y de la Innovación en los Procesos de Enseñanza, doi:10.4067/S0718-50062017000600004, Inf. Tecnol. 10(6), 29-38 (2017)

Martínez, N., Contribución de la formación docente en herramientas web 2.0 en el diseño e implementación de estrategias didácticas en el aula, Tesis de Maestría, Universidad de La Sabana, Centro de tecnología para la academia, Bogotá, Colombia (2016)

MINEDU. Rutas de aprendizaje ¿Qué y cómo aprenden nuestros estudiantes?, Área curricular Matemática, Ministerio de Educación, Lima, Perú (2015)

Morales-Maure, L., O. García-Marimón, A. Torres-Rodríguez y A. Lebrija-Trejos, Habilidades Cognitivas a través de la Estrategia de Aprendizaje Cooperativo y Perfeccionamiento Epistemológico en Matemática de Estudiantes de Primer Año de Universidad, doi:10.4067/S0718-50062018000200045, Inf. Tecnol. 11(2), 45-56 (2018)

Mueller, D. y S. Strohmeier; Design characteristics of virtual learning environments: state of research, doi: http://doi.org/10.1016/j.compedu.2011.06.017, Computers \& Education, 57(4), 2505-2516 (2011)

NCTM. National Council of Teachers of Mathematics, Principles and standards for school mathematics, Reston, VA: NCTM (2000)

OECD. Organization for Economic Cooperation and Development, The PISA 2003, Assessment framework. Mathematics, reading, science and problem solving knowledge and skills, OECD, Paris (2003)

Pérez, Á.l., ¿Competencias o pensamiento práctico? La construcción de los significados de representación y acción, Sinéctica 36, Tlaquepaque enero/junio, México (2011)

Puig, L., Sentido y elaboración del componente de competencia de los modelos teóricos locales en la investigación de la enseñanza y aprendizaje de contenidos matemáticos específicos, ISSN: 1887-3987, PNA, 2(3), $87-107$ (2008)

Ramón, J.A., Enseñanza y aprendizaje de la programación lineal mediada con phpsimplex en la educación secundaria, ISSN: 2448-6469, ALME, 31(1), 912-922 (2018)

Rey, B., Les compétences transversales en question. ESF Editeur: Pedagogies Recherche, París, Francia (1998)

Rodríguez, A., L. Ramirez y F. Basile, Tecnologías y Educación: Su Percepción Social En Santiago de Chile, doi: 10.4067/S0718-50062017000600008, Inf. Tecnol. 10(6), 67-76 (2017)

Rosa, M. y D. Clark, La Etnomatemática como una Perspectiva Metodológica para el Ambiente Virtual de Aprendizaje a Distancia en los Cursos de Formación de Profesores, Asociación Brasileña de Educación Matemática, CEAD/Universidade Federal de Ouro Preto, 12, 99-117 (2013)

Sáenz, C. y A. Lebrija, La formación continua del profesorado de matemáticas: una práctica reflexiva para una enseñanza centrada en el aprendiz, ISSN: 1665-2436, Revista latinoamericana de investigación en matemática educativa, 17(2), 219$244(2014)$

Sánchez, J.C., Análisis de libros de texto de Matemáticas del Ciclo Medio de la Educación General Básica, Tesis doctoral, Dpto. de Métodos de Investigación y Diagnóstico en Educación, Universidad Complutense de Madrid, España (1998)

Stringer, E.T., Action Research, 4ª Ed., Curting University of technology, United States of America (2014)

Tobón, S., J. Pimienta y J. García, Secuencias didácticas: aprendizaje y evaluación de competencias, Printice Hall, México (2010)

Tobón, S., Competencias (2015)

UNESCO. Aportes para la enseñanza de la matemática, Santiago: MIDE UC, por encargo de la Oficina Regional de Educación para América Latina y el Caribe, OREALC/UNESCO, Chile (2016)

UNESCO. Enfoques estratégicos sobre las TIC en educación en América Latina y el Caribe. Oficina Regional de Educación para América Latina y el Caribe (OREALC / UNESCO, Santiago), Chile (2013)

Vilchez, J., La etnomatemática como recurso didáctico en el proceso de aprendizaje de la matemática en zona rural, ISSN: 2448-6469, ALME, 31(1), 567-575 (2018) 